\author{
Marquette University \\ e-Publications@Marquette
}

School of Dentistry Faculty Research and

Publications

Dentistry, School of

2017

\title{
Immunohistochemical Analysis of IL-1 beta in the Discs of Patients with Temporomandibular Joint Dysfunction
}

\author{
Luis Eduardo Almeida \\ Marquette University, luis.almeida@marquette.edu \\ Sean Pierce \\ Marquette University \\ Joseph Zacharias \\ Marquette University \\ William E. Cullinan \\ Marquette University, william.cullinan@marquette.edu \\ Lucia Noronha \\ Pontificia Universidade Catolica do Parana
}

See next page for additional authors

Follow this and additional works at: https://epublications.marquette.edu/dentistry_fac

Part of the Dentistry Commons

\section{Recommended Citation}

Almeida, Luis Eduardo; Pierce, Sean; Zacharias, Joseph; Cullinan, William E.; Noronha, Lucia; Olandoski, Marcia; Tramontina, Vinicius; Loreto, Carla; and Leonardi, Rosalia, "Immunohistochemical Analysis of IL-1 beta in the Discs of Patients with Temporomandibular Joint Dysfunction" (2017). School of Dentistry Faculty Research and Publications. 240.

https://epublications.marquette.edu/dentistry_fac/240 


\section{Authors}

Luis Eduardo Almeida, Sean Pierce, Joseph Zacharias, William E. Cullinan, Lucia Noronha, Marcia Olandoski, Vinicius Tramontina, Carla Loreto, and Rosalia Leonardi 
Marquette University

e-Publications@Marquette

\section{Dentistry Faculty Research and Publications/College of Dentistry}

This paper is NOT THE PUBLISHED VERSION.

Access the published version at the link in the citation below.

Journal of Craniomandibular \& Sleep Practice, Vol. 35, No. 4 (2017): 233-237. DOI. This article is (c) Taylor \& Francis and permission has been granted for this version to appear in $\underline{\text { e- }}$ Publications@Marquette. Taylor \& Francis does not grant permission for this article to be further copied/distributed or hosted elsewhere without the express permission from Taylor \& Francis.

\section{Immunohistochemical Analysis of IL-1 Beta in the Discs of Patients with Temporomandibular Joint Dysfunction}

\section{Luis Eduardo Almeida}

Oral Sciences Department, Oral Surgery, School of Dentistry, Marquette University, Milwaukee, WI Sean Pierce

Oral Sciences Department, Oral Surgery, School of Dentistry, Marquette University, Milwaukee, WI Joseph Zacharias

Oral Sciences Department, Oral Surgery, School of Dentistry, Marquette University, Milwaukee, WI William Cullinan

Biomedical Sciences, College of Health Sciences, Marquette University, Milwaukee, WI Lucia Noronha School of Health and Biosciences, Pontifícia Universidade Católica do Paraná, Curitiba, Brazil Marcia Olandoski

School of Health and Biosciences, Pontifícia Universidade Católica do Paraná, Curitiba, Brazil Vinicius Tramontina 
Periodontics Department, School of Dentistry, Pontifícia Universidade Católica do Paraná, Curitiba, Parana, Brazil

\title{
Carla Loreto
}

Department of Biomedical and Biotechnological Sciences, Human Anatomy and Histology Section, University of Catania, Catania, Italy

\section{Rosalia Leonardi}

Department of Surgical Sciences, University of Catania, Catania, Italy

\begin{abstract}
Purpose: Interleukin-1 beta (IL-1ß) is a cytokine that participates in the regulation of immune responses and inflammatory reactions. It is hypothesized that IL-1 levels may be elevated in patients suffering from temporomandibular joint dysfunction. The purpose of this study was to determine the association of IL-1 $\beta$ expression with TMD using an immunohistochemical approach to evaluate the joint disc.
\end{abstract}

Materials and methods: A total of 39 human temporomandibular joint disc samples were collected, with 31 samples in the test group. Nineteen of the test group samples were from discs of patients with anterior disc displacement with reduction, and 12 of the samples were from patients with anterior disc displacement without reduction. Eight control samples were used in the control group. The samples were immunostained and evaluated on both quantity and intensity of staining.

Results: There was a statistically significant difference $(p<0.05)$ between the control and test groups for both quantity and intensity of staining.

Conclusion: IL-1 $\beta$ plays a role in the inflammatory process and degradation of TMJ discs in patients with TMJ dysfunctions.

\section{Keywords:}

Temporomandibular joint, Immunohistochemistry, IL-1

\section{Introduction}

The temporomandibular joint has the capability to adapt and remodel according to its functional demands. The fibrocartilaginous disc in the TMJ consists of regularly arranged collagen fibers and fibroblast-like cells. Few and scattered, chondrocyte-like cells and fibrochondrocytes can also be appreciated. The disc is responsible for distributing the pressure loads of the TMJ during masticatory function. 1 When the disc is overloaded, an inflammatory process begins, and the disc undergoes remodeling. If the overloading persists for an extended period of time, the disc may undergo structural

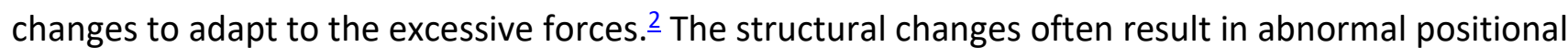
relationships of the articular disc to the mandibular condyle and articular eminence.

These abnormal positional relationships are categorized into a group of temporomandibular joint disorders called internal derangements (ID). $\underline{3}$ There are two common types of ID: anterior disc displacement with reduction (ADDwR) and anterior disc displacement without reduction (ADDwoR). Depending on the stage of derangement, the disc either reseats itself on top of the mandibular condyle when the mouth is opened (ADDwR), or the disc remains in a malposition in relation to the condyle throughout all joint movements (ADDwoR). $\underline{4}$ 
These mechanical joint disturbances cause the joint to undergo structural changes in order to adapt to IDs. Acute macrotrauma is the major cause of disc structural changes. The structural changes are mostly a result of chemical mediators being released in the joint space as well as in the disc itself. $-\underline{5}$ The inflammatory process is a coordinated response against trauma. However, when the initiating inflammatory agent is not removed, the disc's response leads to pathological changes. Interleukin-1 plays an initial and vital role in this inflammatory process and ultimately the cartilage degradation of TMJ discs. $\underline{6}$

In normal, healthy TMJ discs, there are virtually no pro-inflammatory cytokines present. ${ }^{7}$ Soon after TMJ discs are subjected to excessive loads and function, they begin to undergo pathologic changes. These mechanical disturbances produce an imbalance between anabolic and catabolic processes,

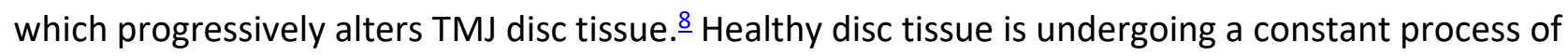
remodeling, whereby collagen and proteoglycan synthesis is balanced by enzymatic extracellular matrix (ECM) degradation. -9 Damage of the TMJ disc is associated with excessive catabolic activity that results from cytokines and proteases. $\underline{10}$ This causes the ECM to remodel. $\underline{11}$ Rates of ECM degradation increase, while ECM synthesis decreases. $\underline{12}$ A number of pro-inflammatory cytokines have been

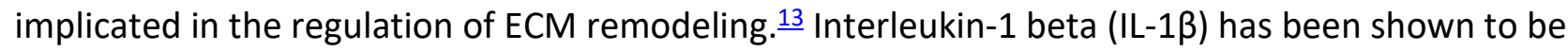
increased in joint degeneration and has been shown to be present in joint synovial fluid, but its presence has not been demonstrated in human TMJ tissue. $\frac{14,15}{1}$ Interleukin- 1 beta is one of the most prevalent and first cytokines produced as a response to the excessive loads placed on the TMJ. $\underline{16}$ The release of IL-1 initiates a cascade of events that ultimately leads to tissue alteration. This cascade alters the ECM by depleting matrix substances such as collagen and proteoglycans.

Although the role of IL-1 $\beta$ in inflammation and joint dysfunction is well known, previous studies have only investigated the presence of IL-1 $\beta$ in animal models, cadavers, or synovial fluid of humans. $\frac{6,7,16}{}$ Based on previous studies, the goal of this study was to histochemically evaluate the expression of IL-1 $\beta$ receptor in the discs from live patients who were diagnosed with TMD. To the authors' knowledge, this is the first study that has used an immunohistochemical approach to characterize the expression of IL-1 $\beta$ in human TMJ disc tissue.

\section{Materials and Methods}

\section{Patients and samples}

A total of 36 disc samples were obtained for this study. The discs were collected from patients treated between 2002 and 2009 at the Hospital Universitario Evangelico de Curitiba. Eight virtually unaffected human discs were included in the control group for the study.

The subjects did not have any of the following: use of orthodontic appliances; chronic usage of antiinflammatory drugs; history of diabetes, hepatitis, or HIV infection; immunosuppressive chemotherapy history of any disease known to severely compromise immune function; current pregnancy or lactation; dentofacial deformity; major jaw trauma previously, TMJ surgery; or previous steroid injection in the TMJ. The subjects completed personal medical history questionnaires and, within a protocol approved by an Institutional Review Board, signed a consent form after being advised of the nature of the study. All patients were asked to complete a pain questionnaire, and an experienced oral and maxillofacial surgeon performed a clinical examination. The clinical examination included palpation 
of the TMJ region, noting the occurrence of painful opening/closing of the mouth, and listening for crepitation.

The patients considered to be affected were treated surgically when they presented painful clinical signs of disc displacement after unsuccessful non-surgical treatment for at least six months. Patients presenting with pain related to muscular spasms were not included in this study. For the complementary exams, all patients received a panoramic radiograph and MRI imaging studies.

The subjects were included in clinical categories, according to the presence or absence of disc displacement.

The test group included a total of 31 samples of discs that were diagnosed with TMJ dysfunctions. This group was separated into anterior disc displacement with reduction $(n=19)$ and ADDwoR $(n=12)$. The control group included a total of eight samples. The discs of these patients were removed for reasons other than TMJ dysfunction. Four of the patients presented with condyle fractures that were confirmed by radiographs and CT scans. Four other control samples were obtained from patients who displayed active condyle hyperplasia. Wedges of the control discs were removed from the posterior regions so that the disc was able to physiologically adapt to the head of the condyle during function.

\section{Interleukin-1 $\beta$ immunohistochemical analysis}

The TMJ discs were deparaffinized with $100 \%$ xylene $(3 \mathrm{~min}$ ) and a 50:50 mix of xylene and ethanol ( $3 \mathrm{~min}$ ). The discs were then rehydrated with absolute ethanol $(2 \times 3 \mathrm{~min}), 90 \%$ ethanol ( $3 \mathrm{~min}), 70 \%$ ethanol (3 $\mathrm{min})$, and distilled water ( $3 \mathrm{~min})$.

The discs were cold incubated for $24 \mathrm{~h}$ with monoclonal IL-1 $\beta$ receptor antibodies (Thermo Fisher Scientific, Rockford, IL, USA) diluted 1:2 000 in a solution of $0.1 \mathrm{M} \mathrm{NaPO}_{4}$ buffer, $0.3 \%$ Triton, and $0.25 \%$ carrageenan. Biotinylated secondary anti-goat/anti-rabbit was diluted 1:100 in the same solution as the primary antibodies and then added to the discs for two hours. Avidin-biotin-peroxidase complex was then added to the slides and allowed to incubate for two hours. The immunoreactions were visualized by incubating the slides with $3.3^{\prime}$ diaminobenzidine chromogen. The slides were once again dehydrated and mounted.

\section{Evaluation of immunohistochemistry}

Interleukin-1 $\beta$ receptor staining was identified as either positive or negative for each disc. Positive staining was defined as the presence of brown chromogen detection, distributed within the cytoplasm or in the immediate lacunar/pericellular space. Two blinded observers evaluated the percentage of immunopositive cells. The observers scored the percentages based on a predetermined scale $(0=<5 \%$; $1=6-30 \% ; 2=31-50 \%, 3=51-75 \% ; 4=76-100 \%)$. Counting was performed at $200 \times$ magnification. The intensity of the staining was also categorized on a four-point predetermined scale $(0=$ no detectable staining, 1 = light staining, 2 = moderate staining, $3=$ strong staining, and $4=$ very strong staining). If the observers' grading disagreed by more than one point, the field of view that was being graded was thrown out and a new field of view was chosen. For each disc, five areas were analyzed for both percentage and intensity of staining. 
Statistical analysis

SPSS version 22.0 (SPSS Institute, Inc., Chicago, IL, USA) was used for the statistical analyses. To analyze the area and density, the average of the results from all available samples of each patient was considered. Means were compared using one-way ANOVA and least significant difference test for multiple comparisons of groups. A value of $p<0.05$ indicated a statistical difference.

\section{Results}

Immunoreactivity for IL-1 $\beta$ expression was confirmed, following IHC. Staining was localized to cells expressing IL-1 $\beta$ receptors. All experimental samples were identified as positively stained. The samples were first analyzed for the percentage of positively stained cells. The experimental groups, ADDWR (Figure 1) and ADDwoR (Figure 2), were both statistically significant when compared to the control group (Figure 3 ). As shown in Table 1 below, IL-1 $\beta$ receptor expression was statistically significant for both ADDwR and ADDwoR $(p<0.001)$. However, there was no significant difference of percentage of positively stained cells between each of the experimental groups $(p=0.063)$ (Table 1$)$.

Figure 1 Anterior disc displacement with reduction.

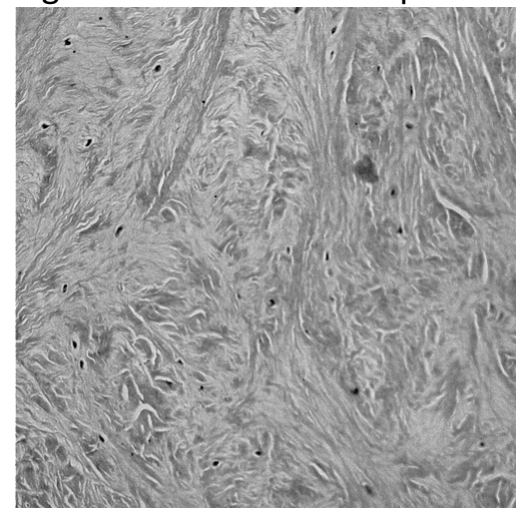

Figure 2 Anterior disc displacement without reduction.

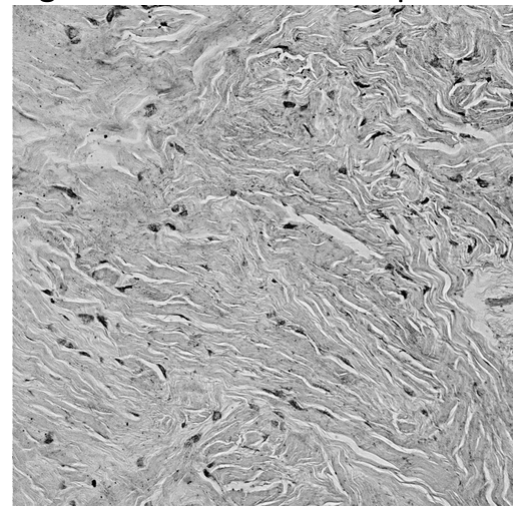

Figure 3 Control. 


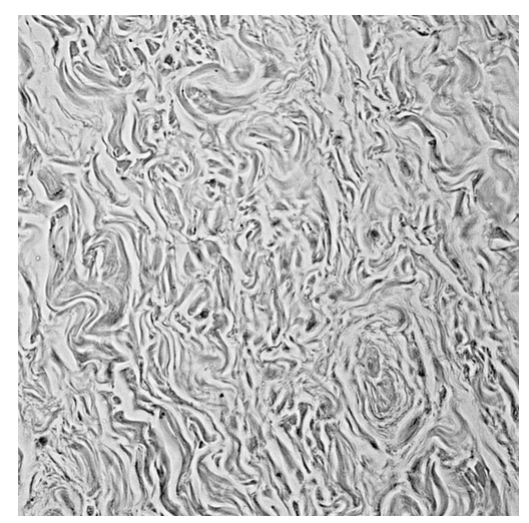

Table 1 Immunoreaction IL-1 $\beta$

\begin{tabular}{|l|l|l|l|l|l|l|l|}
\hline Group & $\begin{array}{l}\text { \# of positively stained } \\
\text { fibroblasts }\end{array}$ & & & & & & $p$ value* \\
\hline & $n$ & Mean & Median & Minimum & Maximum & $\begin{array}{l}\text { Standard } \\
\text { deviation }\end{array}$ & \\
\hline Control & 8 & 0.450 & 0.500 & 0.000 & 1.000 & 0.366 & \\
\hline With red & 19 & 2.147 & 2.400 & 0.200 & 4.000 & 0.882 & \\
\hline Without red & 12 & 2.733 & 2.800 & 1.200 & 4.000 & 0.939 & $<0.001$ \\
\hline
\end{tabular}

Control $\times$ with red: $p<0.001$; Control $\times$ without red: $p<0.001$; With red $\times$ without red: $p=0.063$.

* One-way ANOVA and least significant difference test for multiple comparisons; $p<0.05$.

The intensity of staining for each sample was also analyzed. ADDwR staining intensity was statistically significant compared to the control tissue $(p<0.001)$. Additionally, ADDwoR staining intensity was also statistically significant compared to the control samples $(p<0.001)$. There was no statistical difference between the two experimental groups $(p=0.170)$. The results are displayed below in Table 2 .

Table 2 Intensity of fibroblast staining

\begin{tabular}{|l|l|l|l|l|l|l|l|}
\hline Group & $\begin{array}{l}\text { Intensity of } \\
\text { fibroblast staining }\end{array}$ & & & & & $p$ value* \\
\hline & $n$ & Mean & Median & Minimum & Maximum & $\begin{array}{l}\text { Standard } \\
\text { deviation }\end{array}$ & \\
\hline Control & 8 & 0.700 & 0.600 & 0.000 & 1.600 & 0.545 & \\
\hline With red & 19 & 2.074 & 2.200 & 0.200 & 3.400 & 0.854 & \\
\hline Without red & 12 & 2.483 & 2.600 & 1.200 & 4.000 & 0.824 & $<0.001$ \\
\hline
\end{tabular}

Notes:

Control $\times$ with red: $p<0.001$; Control $\times$ without red: $p<0.001$; With red $\times$ without red: $p=0.170$.

$*$ One-way ANOVA and least significant difference test for multiple comparisons; $p<0.05$.

\section{Discussion}

In the healthy TMJ disc, the rate of synthesis and breakdown of the discal tissue is in equilibrium due to the intricate, but delicate regulation by growth factors and cytokines. Disc cells are likely to play a substantial role in the turnover of the disc matrix. In the diseased state, disc degeneration is associated with a change in the balance between anti- and pro-inflammatory mediators. $\underline{17}$ 
Interleukin-1 $\beta$ has been shown to play an important role in the pathophysiology of joint diseases. $\frac{18}{}$ It has been shown to be present in high concentrations in both the acute and chronic stages of inflammation. $\underline{19}$ Previous disc displacement studies have focused on the synovial fluid and the synovial lining cells. $\frac{7,16,20}{}$ These previous studies have found increased expression of many inflammatory mediators, including interleukins, matrix metalloproteases (MMPs), tumor necrosis factor (TNF), interferon gamma, and transforming growth factor beta (TGF beta). Therefore, the aim of this study was to investigate the expression of IL-1 $\beta$ released from fibroblasts within disc tissue.

The results of this study were similar to the results shown by studies that investigated the expression of IL-1 $\beta$ in synovial fluid. $\underline{5}$ There was a statistically significant difference between the control group and the ADDwR and between the control group and ADDwoR. This suggests that excessive forces on the articular disc initiate similar inflammatory cascades as seen in synovial fluid.

Increased IL-1 beta expression has been shown to not only increase inflammation, but also increase the production and release of other mediators that are responsible for tissue changes and degradation. Multiple studies injected IL-1 $\beta$ into the joint capsules of TMJs in animal models or in tissue samples from humans. They used various laboratory methods to quantify the amounts of secondary mediators released into the TMJ after hours, days, and weeks. Nearly all of the studies correlated an increased release of their test mediator following injection of IL-1 into the joint space. For example, a study found that there was an increased expression of COX-2, IL-6, and MMP-9 following injection of IL-1 into mouse TMJs. They followed the mice and found that the injections led to pathologic development, dysfunction, and related pain in the joints. $\underline{21}$ Additional studies have used similar methods, and they found increased expression of ADAMTS, lumican, fibromodulin, hyaluronan synthase 3, proteoglycan-4, COX 1 and 2, TNF alpha, IFN gamma, and many MMPs. $\underline{8,10,11,14,22-27}$

With these findings in mind, treatment may be targeted at these pathologic mediators in addition to the traditional therapy for disc dysfunction. Pharmacological treatment may be directed at molecules that are responsible for disc degradation or it may be targeted at the mediators responsible for initiating the inflammatory cascade. The findings in this study suggest that IL-1 $\beta$ is one of the initial and most prevalent mediators found, not only in the synovial fluid, but also in the articular discs of the TMJ. Specific pharmacological treatment targeted at IL-1 $\beta$ may be a beneficial treatment option to arrest and prevent further disc degeneration.

\section{Conclusion}

Future analysis of IL-1 $\beta$ and its relationship with previously mentioned downstream mediators within articular discs may contribute to a better understanding of TMJ dysfunction. The findings may also help direct treatment to specific mediators that are responsible for disc remodeling and destruction.

\section{Funding}

This work was supported by the Marquette University - School of dentistry (2015 - Faculty award).

\section{References}

1. Beek M, Aarnts MP, Koolstra JH, Feilzer AJ, Van Eijden TM. Dynamic properties of the human temporomandibular joint disc. J Dent Res. 2001;80:876-80.10.1177/00220345010800030601 
2. Puzas JE, Landeau JM, Tallents R, Albright K, Schwarz EM, Landesberg R. Degradative pathways in tissues of the temporomandibular joint. Cells Tissues Organs. 2001;169:24856.10.1159/000047888

3. Emshoff R, Innerhofer K, Rudisch A, Bertram S. The biological concept of "internal derangement and osteoarthrosis": a diagnostic approach in patients with temporomandibular joint pain? Oral Surg Oral Med Oral Pathol Oral Radiol Endod. 2002;93:39-44.10.1067/moe.2002.117451

4. Hall HD, Wether JR, Gibbs SJ. The mechanism of disc displacement. J Oral Maxillofac Surg. 2001;59:961-2.10.1053/joms.2001.26042

5. Kaneyama K, Segami N, Yoshimura H, Honjo M, Demura N. Increased levels of soluble cytokine receptors in the synovial fluid of temporomandibular joint disorders in relation to joint effusion on magnetic resonance images. J Oral Maxillofac Surg. 2010;68:108893.10.1016/j.joms.2009.10.027

6. Zhang B, Hu J, Man C, Zhu S. Effect of intra-articular administration of interleukin 1 receptor antagonist on cartilage repair in temporomandibular joint. J Craniofac Surg. 2011;22:7114.10.1097/SCS.0b013e31820873c6

7. Kristensen KD, Alstergren $P$, Stoustrup $P$, Kuseler $A$, Herlin $T$, Pedersen TK. Cytokines in healthy temporomandibular joint synovial fluid. J Oral Rehabil. 2014;41:250-56.10.1111/joor.12146

8. Leonardi R, Almeida LE, Loreto C. Lubricin immunohistochemical expression in human temporomandibular joint disc with internal derangement. J Oral Pathol Med. 2011;40:58792.10.1111/jop.2011.40.issue-7

9. Leonardi R, Almeida LE, Trevilatto PC, Loreto C. Occurrence and regional distribution of TRAIL and DR5 on temporomandibular joint discs: comparison of disc derangement with and without reduction. Oral Surg Oral Med Oral Pathol Oral Radiol Endod. 2010;109:24451.10.1016/j.tripleo.2009.09.028

10. Leonardi R, Loreto C, Barbato E, Caltabiano R, Lombardo C, Musumeci G, Lo Muzio L. MMP-13 (collagenase 3 ) localization in human temporomandibular joint discs with internal derangement. Acta Histochem. 2008;110:314-18.10.1016/j.acthis.2007.11.010

11. Leonardi R, Loreto C, Barbato E, Polimeni A, Caltabiano R, Lo Muzio L. A histochemical survey of the human temporomandibular joint disc of patients with internal derangement without reduction. J Craniofac Surg. 2007;18:1429-33.10.1097/scs.0b013e31814fb72a

12. Leonardi R, Musemeci G, Sicurezza E, Loreto C. Lubricin in human temporomandibular joint disc: an immunohistochemical study. Arch Oral Biol. 2012;57:6149.10.1016/j.archoralbio.2011.12.004

13. Leonardi R, Villari L, Bernasconi G, Piacentini C, Baciliero U, Travali S. Cellular S-100 protein immunostaining in human dysfunctional temporomandibular joint discs. Arch Oral Biol. 2000;45:411-8.10.1016/S0003-9969(99)00144-2

14. Loreto C, Leonardi R, Musumeci G, Pannone G, Castorina S. An ex vivo study on immunohistochemical localization of MMP-7 and MMP-9 in temporomandibular joint discs with internal derangement. Eur J Histochem. 2013;57:e12.10.4081/ejh.2013.e12

15. Sicurezza E, Loreto C, Musumeci G, Almeida LE, Rusu M, Grasso C, Leonardi R. Expression of $\beta$ defensin 4 on temporomandibular joint discs with anterior displacement without reduction. J Craniomaxillofac Surg. 2013;41:821-5.10.1016/j.jcms.2013.01.036 
16. Kim YK, Kim SG, Kim BS, Lee JY, Yun PY, Bae JH, et al. Analysis of the cytokine profiles of the synovial fluid in a normal temporomandibular joint: preliminary study. J Craniomaxillofac Surg. 2012;40:e337-41.10.1016/j.jcms.2012.02.002

17. Wang XD, Kou XX, Mao JJ, Gan YH, Zhou YH. Sustained inflammation induces degeneration of the temporomandibular joint. J Dent Res. 2012;91:499-505.10.1177/0022034512441946

18. Suzuki T, Segami N, Kaneyama K, Nishimura M, Nojima T. Specific expression of interleukin-1 in temporomandibular joints with internal derangement: Correlation with clinical findings. Oral Surg Oral Med Oral Pathol Oral Radiol Endod. 1999;88:413-7.10.1016/S1079-2104(99)70054-5

19. Karden R, Ulfgren AK, Reinholt FP, Holmlund A. Inflammatory cell and cytokine patterns in patients with painful clicking and osteoarthritis in the temporomandibular joint. Int J Oral Maillofac Surg. 2003;32:390-6.10.1054/ijom.2002.0357

20. Akamine $\mathrm{Y}$, Kakudo $\mathrm{K}$, Kondo $\mathrm{M}$, Ota $\mathrm{K}$, Muroi $\mathrm{Y}$, Yoshikawa $\mathrm{H}$, et al. Prolonged matrix metalloproteinase-3 high expression after cyclic compressive load on human synovial cells in three-dimensional cultured tissue. Int J Oral Maxillofac Surg. 2012;41:87481.10.1016/j.ijom.2011.10.027

21. Lai YC, Shaftel SS, Miller JN, Tallents RH, Chang Y, Pinkert CA, et al. Intraarticular induction of interleukin-1 $\beta$ expression in the adult mouse, with resultant temporomandibular joint pathologic changes, dysfunction, and pain. Arthritis Rheum. 2006;1184-97.10.1002/(ISSN)15290131

22. Almeida LE, Caporal K, Ambros V, Azevedo M, Noronha L, Leonardi R, et al. Immunohistochemical expression of matrix metalloprotease-2 and matrix metalloprotease-9 in the disks of patients with temporomandibular joint dysfunction. J Oral Pathol Med. 2015;44:75-9.10.1111/jop.2014.44.issue-1

23. Leonardi R, Crimi S, Almeida LE, Pannone G, Musumeci G, Castorina S, et al. ADAMT-4 and ADAMTS-5 expression in human temporomandibular joint discs with internal derangement, correlates with degeneration. J Oral Pathol Med. 2015;44:870-5.

24. Kiga N, Tojyo I, Matsumoto T, Hiraishi Y, Shinohara Y, Makino S, Fujita S. Expression of lumican and fibromodulin following interleukin-1 beta stimulation of disc cells of the human temporomandibular joint. Eur J Histochem. 2011;55:63-7.

25. Matsumot T, Inayama M, Tojyo I, Kiga N, Fujita S. Expression of hyaluronan synthase 3 in deformed human temporomandibular joint discs: in vivo and in vitro studies. Eur J Histochem. 2010;54:237-41.

26. Cheng J, Wang Y, Wang Z, Yang M, Wu Y. Differential regulation of proteoglycan-4 expression by IL-1 $\alpha$ and TGF- $\beta 1$ in rat condylar chondrocytes. Tohoku J Exp Med. 2010;222:2118.10.1620/tjem.222.211

27. Satoh K, Ogura N, Akutsu M, Kuboyama N, Kuyama K, Yamamoto H, et al. Expression of cyclooxygenase- 1 and -2 in IL-1 $\beta$-induced synovitis of the temporomandibular joint. J Oral Pathol Med. 2009;38:584-90.10.1111/jop.2009.38.issue-7 